\title{
Risk of COVID-19 among front-line health-care workers and the general community: a prospective cohort study
}

\author{
Long H Nguyen*, David A Drew*, Mark S Graham*, Amit D Joshi, Chuan-Guo Guo, Wenjie Ma, Raaj S Mehta, Erica T Warner, \\ Daniel R Sikavi, Chun-Han Lo, Sohee Kwon, Mingyang Song, Lorelei A Mucci, Meir J Stampfer, Walter C Willett, A Heather Eliassen, \\ Jaime E Hart, Jorge E Chavarro, Janet W Rich-Edwards, Richard Davies, Joan Capdevila, Karla A Lee, Mary Ni Lochlainn, Thomas Varsavsky, \\ Carole H Sudre, M Jorge Cardoso, Jonathan Wolf, Tim D Spector, Sebastien Ourselint, Claire J Stevest, Andrew T Chant, on behalf of the \\ COronavirus Pandemic Epidemiology Consortium $\neq$
}

\section{Summary}

Background Data for front-line health-care workers and risk of COVID-19 are limited. We sought to assess risk of COVID-19 among front-line health-care workers compared with the general community and the effect of personal protective equipment (PPE) on risk.

Methods We did a prospective, observational cohort study in the UK and the USA of the general community, including front-line health-care workers, using self-reported data from the COVID Symptom Study smartphone application (app) from March 24 (UK) and March 29 (USA) to April 23, 2020. Participants were voluntary users of the app and at first use provided information on demographic factors (including age, sex, race or ethnic background, height and weight, and occupation) and medical history, and subsequently reported any COVID-19 symptoms. We used Cox proportional hazards modelling to estimate multivariate-adjusted hazard ratios (HRs) of our primary outcome, which was a positive COVID-19 test. The COVID Symptom Study app is registered with ClinicalTrials.gov, NCT04331509.

Findings Among 2035395 community individuals and 99795 front-line health-care workers, we recorded 5545 incident reports of a positive COVID-19 test over 34435272 person-days. Compared with the general community, front-line health-care workers were at increased risk for reporting a positive COVID-19 test (adjusted HR 11.61, 95\% CI 10.93-12 33). To account for differences in testing frequency between front-line health-care workers and the general community and possible selection bias, an inverse probability-weighted model was used to adjust for the likelihood of receiving a COVID-19 test (adjusted HR 3·40, 95\% CI 3·37-3·43). Secondary and post-hoc analyses suggested adequacy of PPE, clinical setting, and ethnic background were also important factors.

Interpretation In the UK and the USA, risk of reporting a positive test for COVID-19 was increased among front-line health-care workers. Health-care systems should ensure adequate availability of PPE and develop additional strategies to protect health-care workers from COVID-19, particularly those from Black, Asian, and minority ethnic backgrounds. Additional follow-up of these observational findings is needed.

Funding Zoe Global, Wellcome Trust, Engineering and Physical Sciences Research Council, National Institutes of Health Research, UK Research and Innovation, Alzheimer's Society, National Institutes of Health, National Institute for Occupational Safety and Health, and Massachusetts Consortium on Pathogen Readiness.

Copyright (C) 2020 The Author(s). Published by Elsevier Ltd. This is an Open Access article under the CC BY-NC-ND 4.0 license.

\section{Introduction}

Since its emergence, severe acute respiratory syndrome coronavirus 2 (SARS-CoV-2), which causes COVID-19, has become a global health threat. ${ }^{1}$ As of July 22, 2020, more than 15 million cases of COVID-19 have been documented worldwide, with nearly 618000 deaths. ${ }^{2}$ In the UK and the USA, Black, Asian, and minority ethnic communities have been disproportionately affected. ${ }^{3,4}$ With ongoing community transmission from asymptomatic individuals, disease burden is expected to rise. As a result, there will be an ongoing need for front-line health-care workers in patient-facing roles. Because this work requires close personal exposure to patients with SARS-CoV-2, front-line health-care workers are at high risk of infection, contributing to further spread. ${ }^{5}$ Initial estimates suggest that front-line health-care workers could account for $10-20 \%$ of all diagnoses, ${ }^{6,7}$ with some early evidence that people from Black, Asian, and minority ethnic backgrounds are at higher risk. ${ }^{3}$

Based on experience with other respiratory viruses, consistent use of personal protective equipment (PPE) is important to reduce nosocomial transmission. ${ }^{8}$ Guidelines from the UK and the USA recommend mask use for health-care workers caring for people with COVID-19., ${ }^{9}$ However, global shortages of masks, respirators, face shields, and gowns, caused by surging demand and supply chain disruptions, have led to efforts to conserve PPE through extended use or reuse, and
(1)

Lancet Public Health 2020

5: e475-83

Published Online

July 31,2020

https://doi.org/10.1016/

S2468-2667(20)30164-X

See Comment page e461

${ }^{*}$ Contributed equally as first authors

†Contributed equally

$\ddagger$ Members listed in the appendix Division of Gastroenterology (L H Nguyen MD, D A Drew PhD A D Joshi PhD, C-G Guo MS W Ma ScD, R S Mehta MD, C-H Lo MD, S Kwon MD, $M$ Song ScD, Prof A T Chan MD), Clinical and Translational Epidemiology Unit (ET Warner SCD, L H Nguyen, D A Drew, A D Joshi, C-G Guo, W Ma, R S Mehta, C-H Lo, S Kwon, M Song, Prof A T Chan), Center on Genomics, Vulnerable Populations, and Health Disparities (ET Warner) and Department of Medicine (D R Sikavi MD), Massachusetts General Hospital and Harvard Medical School, Boston, MA, USA; Department of Biostatistics (L H Nguyen, W Ma, R S Mehta), Department of Epidemiology (Prof LA Mucci SCD, Prof M J Stampfer MD Prof W C Willett MD, A $\mathrm{H}$ Eliassen $\mathrm{SCD}$ JE Chavarro MD JW Rich-Edwards ScD, C-H Lo $M$ Song), Department of Nutrition (M Song, Prof W C Willett, J E Chavarro), Department of Environmental Health (J E Hart SCD), and Department of Immunology and Infectious Disease (Prof A T Chan), Harvard TH Chan School of Public Health, Boston, MA, USA; School of Biomedical Engineering and Imaging Sciences (M S Graham PhD, T Varsavsky MSc, C H Sudre PhD, MJ Cardoso PhD 
Prof S Ourselin PhD), and Department of Twin Research and Genetic Epidemiology (K A Lee MBBCh,

$M$ N Lochlainn MBBCh, Prof T D Spector MD,

CJ Steves PhD), King's College London, London, UK; Department of Medicine, Li Ka Shing Faculty of Medicine, University of Hong Kong, Hong Kong Special Administrative Region, China (C-G Guo);

Channing Division of Network Medicine (Prof M J Stampfer, JE Hart, J E Chavarro,

A $\mathrm{H}$ Eliassen), and Division of Women's Health

(JW Rich-Edwards), Department of Medicine, Brigham and Women's Hospital and Harvard Medical School, Boston, MA, USA; Zoe Global, London, UK (R Davies MA, J Capdevila PhD, JWolf MA); Broad Institute of Massachusetts Institute of Technology and Harvard, Cambridge, MA, USA (Prof A T Chan); and Massachusetts Consortium on Pathogen Readiness, Cambridge, MA, USA (Prof A T Chan)

Correspondence to: Prof Andrew T Chan, Massachusetts General Hospital and Harvard Medical School, Boston, MA 02114, USA achan@mgh.harvard.edu See Online for appendix
Research in context

\section{Evidence before this study}

We searched PubMed for articles published between Jan 1 and June 30, 2020, with the terms "covid-19", "healthcare workers", and "personal protective equipment". We did not restrict our search by language or type of publication. The prolonged course of the COVID-19 pandemic, coupled with sustained challenges supplying adequate personal protective equipment (PPE) for front-line health-care workers, have strained global health-care systems in an unprecedented fashion. Despite growing awareness of this problem, there are few studies to inform policy makers on the risk of COVID-19 among health-care workers and the effect of PPE on disease burden. Previous reports of infections in health-care workers are based on cross-sectional data with limited information on individual-level risk factors. Our PubMed search yielded no population-scale investigations.

\section{Added value of this study}

We did a prospective observational study of 2135190 individuals, comprised of front-line health-care workers and the general community who were voluntary

disinfection protocols have been developed, for which scientific consensus on best practice is scarce. ${ }^{11-13}$

Although addressing the needs of front-line health-care workers during the COVID-19 pandemic is a high priority, ${ }^{6,14}$ data to inform such efforts are scarce, and particularly so among Black, Asian, and minority ethnic communities. Thus, we did a prospective populationbased study using data from a smartphone-based application (app) to investigate the risk of testing positive for COVID-19, the risk of developing symptoms associated with SARS-CoV-2 infection, or both, among individuals in the UK and the USA.

\section{Methods}

\section{Study design and participants}

COVID Symptom Study (previously known as COVID Symptom Tracker) is a free smartphone app developed by Zoe Global (London, UK) in collaboration with Massachusetts General Hospital (Boston, MA, USA) and King's College London (London, UK). It offers participants a guided interface to report baseline demographic information and comorbidities, daily information on symptoms, and COVID-19 testing. Participants are encouraged to log daily, even when asymptomatic, for longitudinal collection of incident symptoms and COVID-19 testing results. The app was launched in the UK on March 24, 2020, and the USA on March 29, 2020.

We did a prospective, observational cohort study using the COVID Symptom Study app. Participants were recruited through social media outreach and invitations from the investigators of long-running cohort studies to their volunteers (appendix p 3). At enrolment, participants consented to use of information for research and users of the COVID Symptom Study smartphone application (app). From self-reported data obtained via this app, we found that front-line health-care workers had at least a threefold increased risk of COVID-19. Compared with front-line health-care workers who reported adequate availability of PPE, those with inadequate PPE had an increase in risk. However, adequate availability of PPE did not seem to completely reduce risk among health-care workers caring for patients with COVID-19. We also found that Black, Asian, and minority ethnic health-care workers might be disproportionately affected.

\section{Implications of all the available evidence}

Front-line health-care workers, particularly those who are from Black, Asian, and minority ethnic backgrounds, could be at substantially greater risk of COVID-19. Health-care systems should ensure adequate availability of PPE and develop additional strategies to protect health-care workers from COVID-19.

agreed to applicable privacy policies and terms of use. Our study was approved by the Partners Human Research Committee (protocol 2020P000909) and King's College London ethics committee (REMAS ID 18210, LRS-19/20-18210).

\section{Procedures}

Information obtained through the COVID Symptom Study app has been described in detail. ${ }^{15}$ Briefly, on first use, participants were asked to provide demographic factors and were questioned separately about a series of COVID-19 risk factors (appendix pp 4-8). At enrolment and with daily reminders, participants were asked if they felt physically normal, and if they reported not feeling well they were asked about their symptoms (appendix p 11). Participants were also asked if they had been tested for COVID-19 and the results (none, negative, pending, or positive). Our primary outcome was a report of a positive COVID-19 test. Follow-up started when participants first reported on the COVID Symptom Study app and continued until a report of a positive COVID-19 test or the time of last data entry, whichever occurred first.

Participants were also asked if they worked in health care and, if yes, whether they had direct patient contact. For our primary analysis, we defined front-line healthcare workers as participants who reported direct patient contact. Prespecified secondary analyses among frontline health-care workers investigated PPE availability and contact with patients with COVID-19, as well as the primary site of clinical practice. A post-hoc analysis among front-line health-care workers assessed the effect of race and ethnicity. Beginning March 29, 2020, in an updated version of the app used by $84 \%$ of participating health-care workers, we included mandatory questions 
about availability of PPE for participants who identified as a front-line health-care worker. Among these individuals, we asked whether they cared for patients with suspected or documented SARS-CoV-2 infection and the frequency with which they used PPE (always, sometimes, or never). We asked if they had enough PPE when needed, if they had to reuse PPE, or if they did not have enough because of shortages. We classified PPE as adequate if they never required PPE or if they reported always having the PPE they needed. We classified PPE as inadequate if they reported they did not have enough PPE or if it was not available. We also asked health-care workers to report the site of their patient care (inpatient, nursing home, outpatient, home health, ambulatory clinic, or other).

\section{Statistical analysis}

We used standard calculations to determine the minimum detectable hazard ratio (HR) for our primary categorical exposure (health-care worker status) and risk of reporting a positive COVID-19 test with $80 \%$ power. ${ }^{16}$

We used Cox proportional hazards modelling stratified by age, date, and country to estimate multivariableadjusted HRs and 95\% CIs. Covariates were selected a priori based on putative risk factors, including sex (male or female), race or ethnic origin (non-Hispanic white, Hispanic or Latinx, Black, Asian, or more than one or other), history of diabetes (yes or no), heart disease (yes or no), lung disease (yes or no), kidney disease (yes or no), current smoking status (yes or no), and bodymass index $\left(17.0-19.9 \mathrm{~kg} / \mathrm{m}^{2}, 20.0-24.9 \mathrm{~kg} / \mathrm{m}^{2}\right.$, $25 \cdot 0-29.9 \mathrm{~kg} / \mathrm{m}^{2}$, and $\left.\geq 30 \cdot 0 \mathrm{~kg} / \mathrm{m}^{2}\right)$. Data imputation replaced no more than $5 \%$ of missing values for a given metadatum, with numerical values replaced with the median and categorical variables imputed using the mode.

Because our primary outcome (positive COVID-19 test) required a participant to receive a test, we did several prespecified secondary analyses. First, we tested a symptom-based classifier predictive of positive COVID-19 testing. ${ }^{17}$ Briefly, using logistic regression and symptoms preceding testing, we have previously described that loss of smell or taste, fatigue, persistent cough, and loss of appetite predicted COVID-19 positivity with high specificity (appendix p 2). Second, to account for countryspecific predictors of obtaining testing, we did separate inverse probability weighting in the UK and the USA as a function of demographic and clinical factors, followed by inverse probability-weighted Cox proportional hazards modelling stratified by age and date with additional adjustment for the covariates used in previous models (appendix p 2). To assess factors associated with PPE adequacy, work in especially high-risk clinical settings, or greater exposure to patients with COVID-19 (including a post-hoc analysis of race and ethnicity), we used multivariable logistic regression models to estimate adjusted odds ratios (ORs) and 95\% CIs. Two-sided p values less than 0.05 were considered statistically significant. All analyses were done using $R$ version 3.6.1.

The COVID Symptom Study app is registered with ClinicalTrials.gov, NCT04331509.

\section{Role of the funding source}

Zoe Global developed the app for data collection. The funders had no role in study design, data analysis, data interpretation, or writing of the report. LHN, DAD, MSG, SO, CJS, and ATC had access to raw data. The corresponding author had full access to all data in the study and had final responsibility for the decision to submit for publication.

\section{Results}

Between March 24 and April 23, 2020, we enrolled 2810103 consecutive users of the COVID Symptom Study app to our study. 2627695 participants in the UK and 182408 in the USA provided baseline information about feeling physically normal or having symptoms (appendix p 14). 134885 (4.8\%) participants reported being a front-line health-care worker. The prevalence of COVID-19 was 2747 cases per 100000 front-line healthcare workers compared with 242 cases per 100000 people in the general community (figure). The highest infection rates were reported in the US states New York, New Jersey, and Louisiana and in areas around London and the Midlands in the UK.

After excluding 670298 participants with less than $24 \mathrm{~h}$ of follow-up and 4615 individuals who reported a positive COVID-19 test at baseline, we included 2135190 participants in our prospective inception cohort, of whom 99795 $(4.7 \%)$ identified as front-line health-care workers (appendix p 14). Based on this cohort, we had $80 \%$ power to detect a minimum HR of $1 \cdot 16$ for risk of reporting a positive COVID-19 test between health-care workers and the general community. In this cohort, we recorded 24.4 million entries, or 11.5 logs per participant, with median follow-up of $18 \cdot 9$ days (IQR 5.1-26.1). Median age was 44 years (IQR 32-57). Compared with the general community, front-line health-care workers were more frequently female, had a slightly higher prevalence of body-mass index $30.0 \mathrm{~kg} / \mathrm{m}^{2}$ or higher, were slightly more likely to smoke (particularly in the UK), and were more likely to use non-steroidal anti-inflammatory drugs (table 1; appendix pp 9-10). At baseline, 20.2\% of frontline health-care workers reported at least one symptom associated with SARS-CoV-2 infection compared with $14.4 \%$ of the general population; fatigue, loss of smell or taste, and hoarse voice were especially frequent (appendix $\mathrm{p}$ 11). When comparing health-care workers who were asked about PPE use with those who were not asked, no difference was noted in baseline factors including age (median 45 years vs 40 years), female sex ( $81 \%$ vs $82 \%$ ), or body-mass index (median $25.9 \mathrm{~kg} / \mathrm{m}^{2}$ vs $25.7 \mathrm{~kg} / \mathrm{m}^{2}$ ).

We recorded 5545 incident reports of positive COVID-19 testing over 34435272 person-days. In the UK, $1.1 \%$ of
For more on $R$ see https://r-project.org 


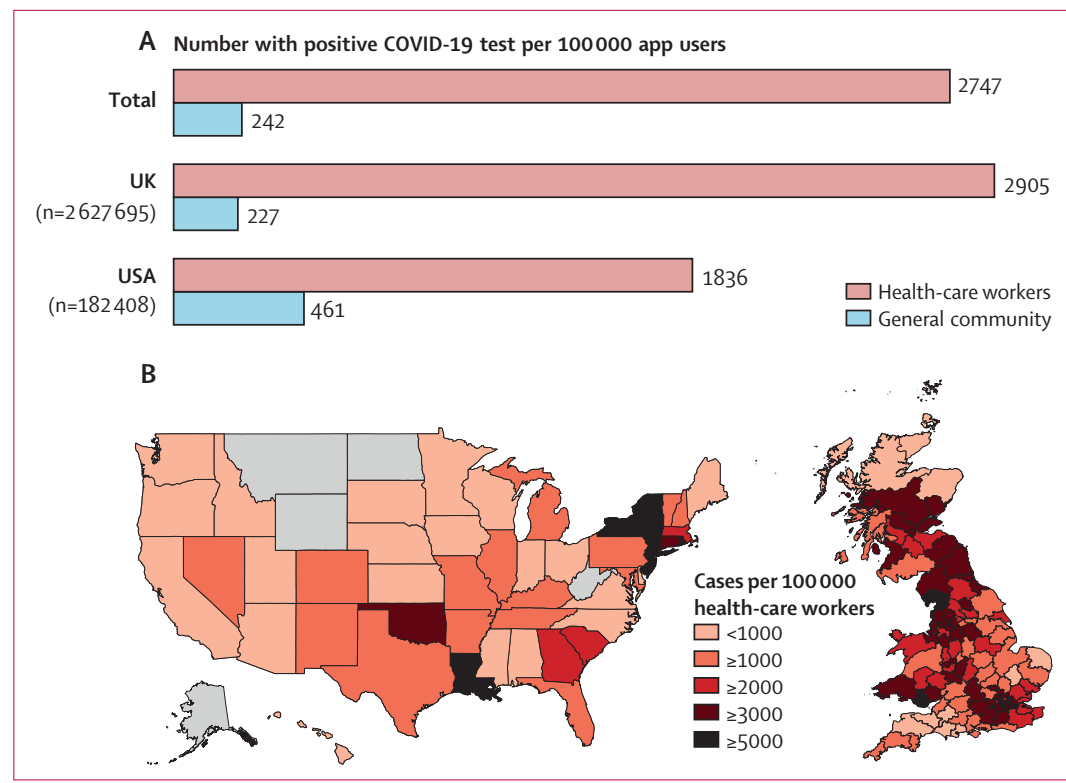

Figure: Risk of testing positive for COVID-19 among front-line health-care workers

(A) Between March 24 and April 23, 2020, considerable disparities were noted in prevalence of a positive COVID-19 test among front-line health-care workers compared with the general community, in both the UK and the USA. (B) Prevalence of a positive COVID-19 test reported by front-line health-care workers in the UK and the USA. Regions in grey did not have sufficient data for analysis. app=COVID-19 Symptom Study smartphone application.

\begin{tabular}{|c|c|c|}
\hline & $\begin{array}{l}\text { Front-line } \\
\text { health-care } \\
\text { workers } \\
(\mathrm{n}=99795)\end{array}$ & $\begin{array}{l}\text { General } \\
\text { community } \\
(n=2035395)\end{array}$ \\
\hline \multicolumn{3}{|c|}{ (Continued from previous column) } \\
\hline Body-mass index $\left(\mathrm{kg} / \mathrm{m}^{2}\right)$ & $25 \cdot 8(22 \cdot 8-30 \cdot 2)$ & $25 \cdot 3(22 \cdot 5-29 \cdot 1)$ \\
\hline $17 \cdot 0-19 \cdot 9$ & $5 \cdot 8 \%$ & $8 \cdot 3 \%$ \\
\hline $20 \cdot 0-24 \cdot 9$ & $38 \cdot 1 \%$ & $39 \cdot 2 \%$ \\
\hline $25 \cdot 0-29 \cdot 9$ & $30 \cdot 1 \%$ & $31 \cdot 5 \%$ \\
\hline$\geq 30.0$ & $25.9 \%$ & $21 \cdot 1 \%$ \\
\hline $\begin{array}{l}\text { Missing data for body-mass } \\
\text { index }\end{array}$ & $0.5 \%$ & $0.5 \%$ \\
\hline \multicolumn{3}{|l|}{ Comorbidities } \\
\hline Diabetes & $2 \cdot 5 \%$ & $3.1 \%$ \\
\hline Heart disease & $1.6 \%$ & $2 \cdot 4 \%$ \\
\hline Lung disease & $13 \cdot 1 \%$ & $12 \cdot 2 \%$ \\
\hline Kidney disease & $0.6 \%$ & $0.7 \%$ \\
\hline Cancer & $0.5 \%$ & $1 \cdot 3 \%$ \\
\hline Missing data for cancer & $0.3 \%$ & $0.3 \%$ \\
\hline Pregnant (\% of females) & $0.9 \%$ & $1.0 \%$ \\
\hline \multicolumn{3}{|l|}{ Medication use } \\
\hline $\begin{array}{l}\text { Non-steroidal } \\
\text { anti-inflammatory drugs }\end{array}$ & $8 \cdot 2 \%$ & $6 \cdot 1 \%$ \\
\hline Immunosuppressants & $2.5 \%$ & $3 \cdot 2 \%$ \\
\hline $\begin{array}{l}\text { Chemotherapy or } \\
\text { immunotherapy }\end{array}$ & $0.1 \%$ & $0.3 \%$ \\
\hline $\begin{array}{l}\text { Angiotensin-converting } \\
\text { enzyme inhibitor }\end{array}$ & $5.0 \%$ & $4 \cdot 9 \%$ \\
\hline $\begin{array}{l}\text { Missing data for } \\
\text { angiotensin-converting } \\
\text { enzyme inhibitor }\end{array}$ & $10 \cdot 1 \%$ & $4 \cdot 3 \%$ \\
\hline \multicolumn{3}{|l|}{ Current smoking } \\
\hline Yes & $10 \cdot 2 \%$ & $8.5 \%$ \\
\hline $\begin{array}{l}\text { Missing data for smoking } \\
\text { status }\end{array}$ & $0.2 \%$ & $0.1 \%$ \\
\hline \multicolumn{3}{|c|}{$\begin{array}{l}\text { Data are \% or median (IQR). \% are calculated based on the total number of } \\
\text { participants with available data. Polytomous variables might not add up to } 100 \% \\
\text { because of rounding. Questions about history of cancer, angiotensin-converting } \\
\text { enzyme inhibitor use, and smoking status have been asked since launch in the USA } \\
\text { and March 29, 2020, in the UK; questions about race and ethnic origin were asked } \\
\text { since April 17, 2020, in both the UK and the USA. Percentages within each category } \\
\text { are based on the total population responding when the question was first asked. } \\
\text { *Non-Hispanic white defined as UK White, US White, and no designation of other } \\
\text { race or ethnic origin. Hispanic or Latinx designated as Hispanic and Latino. Black } \\
\text { defined as UK Black, Black British, US Black, and African American. White defined as } \\
\text { UKWhite and US White. Asian defined as UK Asian, Asian British, UK Chinese, Chinese } \\
\text { British, US Asian, and US Native Hawaiian or other Pacific Islander. More than one or } \\
\text { other defined as UK mixed race White and Black or Black British UK, mixed race other, } \\
\text { UK Middle Eastern or Middle Eastern British, US American Indian or Alaska Native, } \\
\text { other, and denoted more than one race. }\end{array}$} \\
\hline
\end{tabular}

health-care workers reported being tested compared with $0 \cdot 2 \%$ of the general community (health-care workers to community testing ratio $5 \cdot 5)$, whereas $4 \cdot 1 \%$ of US healthcare workers were tested versus $1 \cdot 1 \%$ of the general community (testing ratio $3 \cdot 7)$. Compared with the general community, front-line health-care workers had a twelvefold increase in risk of a positive test after multivariable 


\begin{tabular}{|c|c|c|c|c|c|}
\hline & Event/person-days & $\begin{array}{l}\text { Incidence } \\
\text { (30-day) }\end{array}$ & $\begin{array}{l}\text { Age-adjusted } \\
\text { hazard ratio }(95 \% \mathrm{Cl})\end{array}$ & $\begin{array}{l}\text { Multivariate- } \\
\text { adjusted hazard ratio } \\
(95 \% \mathrm{Cl})\end{array}$ & $\begin{array}{l}\text { Inverse probability- } \\
\text { weighted hazard } \\
\text { ratio }(95 \% \mathrm{Cl})\end{array}$ \\
\hline \multicolumn{6}{|l|}{ Overall (primary analysis) } \\
\hline General community & $3623 / 32980571$ & $0.33 \%$ & 1 (ref) & 1 (ref) & 1 (ref) \\
\hline Front-line health-care worker & $1922 / 1454701$ & $3 \cdot 96 \%$ & $11.68(10 \cdot 99-12 \cdot 40)$ & $11 \cdot 61(10 \cdot 93-12 \cdot 33)$ & $3 \cdot 40(3 \cdot 37-3 \cdot 43)$ \\
\hline \multicolumn{6}{|c|}{ According to race or ethnic origin (post-hoc analysis) } \\
\hline Non-Hispanic white, general community & $1498 / 23941092$ & $0.19 \%$ & 1 (ref) & 1 (ref) & 1 (ref) \\
\hline $\begin{array}{l}\text { Black, Asian, and minority ethnic, general } \\
\text { community }\end{array}$ & $227 / 1362956$ & $0 \cdot 50 \%$ & $2 \cdot 49(2 \cdot 16-2 \cdot 86)$ & $2 \cdot 51(2 \cdot 18-2 \cdot 89)$ & $1 \cdot 74(1 \cdot 71-1 \cdot 77)$ \\
\hline $\begin{array}{l}\text { Non-Hispanic white, front-line } \\
\text { health-care worker }\end{array}$ & $726 / 935860$ & $2 \cdot 33 \%$ & $12 \cdot 47(11 \cdot 33-13 \cdot 72)$ & $12 \cdot 58(11 \cdot 42-13 \cdot 86)$ & $3.52(3.48-3 \cdot 56)$ \\
\hline $\begin{array}{l}\text { Black, Asian, and minority ethnic, } \\
\text { front-line health-care worker }\end{array}$ & $98 / 72556$ & $4.05 \%$ & $21.68(17 \cdot 61-26 \cdot 68)$ & $21 \cdot 88(17 \cdot 78-26 \cdot 94)$ & $4 \cdot 88(4 \cdot 76-5 \cdot 01)$ \\
\hline \multicolumn{6}{|c|}{$\begin{array}{l}\text { All models were stratified by } 5 \text {-year age group, calendar date at study entry, and country. Multivariate risk factor models were adjusted for sex (male or female), history of } \\
\text { diabetes (yes or no), heart disease (yes or no), lung disease (yes or no), kidney disease (yes or no), current smoking (yes or no), and body-mass index (17.0-19.9 kg/m², } \\
20 \cdot 0-24.9 \mathrm{~kg} / \mathrm{m}^{2}, 25 \cdot 0-29 \cdot 9 \mathrm{~kg} / \mathrm{m}^{2} \text {, and } \geq 30 \mathrm{~kg} / \mathrm{m}^{2} \text { ). Black, Asian, and minority ethnic was defined among individuals who had race or ethnicity information and did not } \\
\text { identify as non-Hispanic white. }\end{array}$} \\
\hline
\end{tabular}

adjustment (adjusted HR 11.61, 95\% CI 10.93-12.33; table 2; appendix $\mathrm{p} 15)$. The association seemed stronger in the UK (adjusted HR 12.52, 95\% CI 11.77-13.31) compared with the USA $\left(2 \cdot 80,2 \cdot 09-3 \cdot 75\right.$; $\mathrm{p}_{\text {difference }}<0 \cdot 0001$; appendix $\mathrm{p}$ 12).

We considered the possibility that noted differences could be related to testing eligibility. A multivariableadjusted Cox proportional hazards model with inverse probability weighting for predictors of testing also showed a higher risk of infection among front-line health-care workers (adjusted HR 3.40, 95\% CI 3.37-3.43; table 2), which was higher in the UK $(3 \cdot 43,3 \cdot 18-3 \cdot 69)$ than in the USA $(1 \cdot 97,1 \cdot 36-2 \cdot 85$; $\mathrm{p}_{\text {difference }}<0.0001$; appendix $\mathrm{p}$ 12). In a prespecified secondary analysis, a validated model was used based on a combination of symptoms predictive of COVID-19 infection. ${ }^{16}$ Compared with the general community, health-care workers initially free of symptoms had an increased risk of predicted COVID-19 (adjusted HR 2.05, $95 \%$ CI 1.99-2.10), which was higher in the UK $(2 \cdot 09,2 \cdot 02-2 \cdot 15)$ than in the USA $(1 \cdot 31,1 \cdot 14-1 \cdot 51$; $\left.\mathrm{p}_{\text {difference }}<0 \cdot 0001\right)$.

In a post-hoc analysis, compared with individuals in the general community from a non-Hispanic white ethnic background, the risk for a positive COVID-19 test was increased for individuals from Black, Asian, and minority ethnic backgrounds in the general community (adjusted HR 2.51, 95\% CI 2.18-2.89), for Black, Asian, and minority ethnic health-care workers $(21.88$, 17.78-26.94), and for non-Hispanic white health-care workers $(12 \cdot 58,11 \cdot 42-13 \cdot 86$; table 2$)$. In post-hoc analyses, the association of race and health-care worker status with risk of COVID-19 was assessed. Black, Asian, and minority ethnic health-care workers had an increased risk of COVID-19 (adjusted HR 1.81, 95\% CI 1.45-2·24) compared with non-Hispanic white health-care workers.

\begin{tabular}{|c|c|c|c|}
\hline & Adequate PPE & Reused PPE & Inadequate PPE \\
\hline \multicolumn{4}{|l|}{ Overall } \\
\hline Event/person-days & $592 / 332901$ & $146 / 80728$ & $157 / 60916$ \\
\hline Unadjusted hazard ratio $(95 \% \mathrm{Cl})$ & 1 (ref) & $1.46(1 \cdot 21-1 \cdot 76)$ & $1 \cdot 32(1 \cdot 10-1 \cdot 57)$ \\
\hline $\begin{array}{l}\text { Multivariate-adjusted hazard ratio } \\
(95 \% \mathrm{Cl})\end{array}$ & 1 (ref) & $1 \cdot 46(1 \cdot 21-1 \cdot 76)$ & $1 \cdot 31(1 \cdot 10-1 \cdot 56)$ \\
\hline \multicolumn{4}{|c|}{ No exposure to patients with COVID-19 } \\
\hline Event/person-days & $186 / 227654$ & 19/37599 & $48 / 35159$ \\
\hline Unadjusted hazard ratio $(95 \% \mathrm{Cl})$ & 1 (ref) & $0.96(0.60-1.55)$ & $1.53(1.11-2 \cdot 11)$ \\
\hline $\begin{array}{l}\text { Multivariate-adjusted hazard ratio } \\
(95 \% \mathrm{Cl})\end{array}$ & 1 (ref) & $0.95(0.59-1.54)$ & $1 \cdot 52(1 \cdot 10-2 \cdot 09)$ \\
\hline \multicolumn{4}{|c|}{ Exposure to patients with suspected COVID-19 } \\
\hline Event/person-days & $126 / 54676$ & $36 / 19378$ & $26 / 14083$ \\
\hline Unadjusted hazard ratio $(95 \% \mathrm{Cl})$ & $2.40(1.91-3.02)$ & $3 \cdot 23(2 \cdot 24-4 \cdot 66)$ & $1.87(1.24-2 \cdot 83)$ \\
\hline $\begin{array}{l}\text { Multivariate-adjusted hazard ratio } \\
(95 \% \mathrm{Cl})\end{array}$ & $2 \cdot 39(1.90-3 \cdot 00)$ & $3 \cdot 20(2 \cdot 22-4 \cdot 61)$ & $1.83(1.21-2 \cdot 78)$ \\
\hline \multicolumn{4}{|c|}{ Exposure to patients with documented COVID-19 } \\
\hline Event/person-days & $280 / 50571$ & $91 / 23751$ & $83 / 11675$ \\
\hline Unadjusted hazard ratio $(95 \% \mathrm{Cl})$ & $4.93(4.07-5.97)$ & $5 \cdot 12(3.94-6.64)$ & $5 \cdot 95(4 \cdot 57-7 \cdot 76)$ \\
\hline $\begin{array}{l}\text { Multivariate-adjusted hazard ratio } \\
(95 \% \mathrm{Cl})\end{array}$ & $4.83(3.99-5.85)$ & $5.06(3.90-6 \cdot 57)$ & $5 \cdot 91(4 \cdot 53-7 \cdot 71)$ \\
\hline \multicolumn{4}{|c|}{$\begin{array}{l}\text { All models were stratified by } 5 \text {-year age group, calendar date at study entry, and country. Multivariate risk factor } \\
\text { models were adjusted for sex (male or female), history of diabetes (yes or no), heart disease (yes or no), lung disease } \\
\text { (yes or no), kidney disease (yes or no), current smoking (yes or no), and body-mass index (17.0-19.9 kg/m², } \\
20 \cdot 0-24 \cdot 9 \mathrm{~kg} / \mathrm{m}^{2}, 25 \cdot 0-29 \cdot 9 \mathrm{~kg} / \mathrm{m}^{2} \text {, and } \geq 30.0 \mathrm{~kg} / \mathrm{m}^{2} \text { ). PPE=personal protective equipment. }\end{array}$} \\
\hline
\end{tabular}

Risk estimates were similar among male (adjusted HR $14 \cdot 02$, 95\% CI 12.38-15.82) compared with female $(11 \cdot 27,10 \cdot 53-12 \cdot 14)$ front-line health-care workers.

Among front-line health-care workers, availability and use of PPE, COVID-19 patient exposures, and subsequent risk for testing positive were assessed in prespecified analyses. Compared with health-care workers who reported adequate PPE, front-line health-care workers 
reporting PPE reuse had an increased risk of a positive COVID-19 test (adjusted HR 1.46, 95\% CI 1.21-1.76), with inadequate PPE associated with a comparable increase in risk after multivariable adjustment $(1 \cdot 31$, $1 \cdot 10-1 \cdot 56$; table 3 ).

In a prespecified secondary analysis, front-line healthcare workers with inadequate PPE caring for patients with documented COVID-19 had an increased risk for COVID-19 after multivariable adjustment (adjusted HR 5.91, 95\% CI 4.53-7.71) compared with those with adequate PPE not caring for patients with suspected or documented COVID-19 (table 3). Risk for front-line health-care workers reusing PPE and exposed to patients with documented COVID-19 was also increased (adjusted HR 5.06, 95\% CI 3.90-6.57). Notably, even among

\begin{tabular}{|c|c|c|}
\hline & $\begin{array}{l}\text { Health-care workers } \\
\text { reporting reuse of or } \\
\text { inadequate PPE }\end{array}$ & $\begin{array}{l}\text { Multivariate-adjusted odds } \\
\text { ratio }(95 \% \mathrm{Cl})\end{array}$ \\
\hline \multicolumn{3}{|l|}{ Overall } \\
\hline $\begin{array}{l}\text { Non-Hispanic white, front-line health-care } \\
\text { worker }\end{array}$ & $27 \cdot 7 \%$ & 1 (ref) \\
\hline $\begin{array}{l}\text { Black, Asian, and minority ethnic, front-line } \\
\text { health-care worker }\end{array}$ & $36 \cdot 7 \%$ & $1.49(1.36-1.63)$ \\
\hline \multicolumn{3}{|l|}{ According to racial or ethnic subgroup* } \\
\hline Non-Hispanic white` & $27 \cdot 7 \%$ & 1 (ref) \\
\hline Hispanic or Latinx & $49 \cdot 6 \%$ & $2.64(2.03-3 \cdot 45)$ \\
\hline Black & $33 \cdot 5 \%$ & $1.30(1.02-1.65)$ \\
\hline Asian & $35 \cdot 6 \%$ & $1.42(1.24-1 \cdot 63)$ \\
\hline More than one race or other race & $34 \cdot 7 \%$ & $1 \cdot 33(1 \cdot 12-1 \cdot 57)$ \\
\hline
\end{tabular}

Multivariate risk factor models were adjusted for 5-year age group, sex, and exposure to patients with COVID-19 (none, suspected, and documented). Black, Asian, and minority ethnic was defined among individuals who had race or ethnicity information and did not identify as non-Hispanic white. PPE=personal protective equipment. *Non-Hispanic white defined as UK White, US White, and no designation of other race or ethnic origin. Hispanic or Latinx designated as Hispanic and Latino. Black defined as UK Black, Black British, US Black, and African American. White defined as UK White and US White. Asian defined as UK Asian, Asian British, UK Chinese, Chinese British, US Asian, and US Native Hawaiian or other Pacific Islander. More than one or other defined as UK mixed race White and Black or Black British UK, mixed race other, UK Middle Eastern or Middle Eastern British, US American Indian or Alaska Native, other, and denoted more than one race.

Table 4: Risk of reporting PPE inadequacy or reuse among front-line health-care workers, according to race or ethnic origin (post-hoc analysis) front-line health-care workers reporting adequate PPE, the risk for COVID-19 was increased for those caring for patients with suspected COVID-19 (adjusted HR 2.39, 95\% CI 1.90-3.00) and for those caring for patients with documented COVID-19 (4.83, 3.99-5.85), compared with health-care workers who did not care for either group (table 3).

In a post-hoc analysis, differences were noted in PPE adequacy according to race and ethnicity, with non-white health-care workers more frequently reporting reuse of or inadequate access to PPE, even after adjusting for exposure to patients with COVID-19 (adjusted OR 1.49, 95\% CI 1.36-1.63; table 4).

In a prespecified secondary analysis, risk of COVID-19 by practice location was assessed. Compared with risk for the general community, risk for front-line health-care workers was increased in all health-care settings, but was highest for those working in inpatient settings (adjusted HR 24.30, 95\% CI 21.83-27.06) and nursing homes $(16 \cdot 24,13 \cdot 39-19 \cdot 70$; table 5). Notably, health-care workers in nursing homes most frequently (16.9\%) reported inadequate PPE, whereas inpatient providers reported reuse of PPE most often (23.7\%; table 5). In a post-hoc analysis, compared with non-Hispanic white health-care workers, Black, Asian, and minority ethnic health-care workers were more likely to work in higher risk clinical settings, including inpatient hospital or nursing homes (adjusted OR 1.13, 95\% CI 1.03-1.23) and to care for patients with suspected or documented COVID-19 $(1 \cdot 20,1 \cdot 09-1 \cdot 30)$. These noted differences were most pronounced among Black health-care workers (appendix p 13).

\section{Discussion}

Among 2135190 people in the UK and USA using the COVID-19 Symptom Study app between March 24 and April 23, 2020, we noted that front-line health-care workers had at least a threefold increased risk of reporting a positive COVID-19 test and predicted COVID-19 infection, compared with the general community, even after

\begin{tabular}{|c|c|c|c|c|c|c|}
\hline & $\begin{array}{l}\text { Event/ } \\
\text { person-days }\end{array}$ & $\begin{array}{l}\text { Incidence } \\
\text { (30-day) }\end{array}$ & $\begin{array}{l}\text { Age-adjusted hazard } \\
\text { ratio }(95 \% \mathrm{Cl})\end{array}$ & $\begin{array}{l}\text { Multivariate-adjusted } \\
\text { hazard ratio }(95 \% \mathrm{Cl})\end{array}$ & $\begin{array}{l}\text { Health-care } \\
\text { workers reporting } \\
\text { reuse of PPE }\end{array}$ & $\begin{array}{l}\text { Health-care } \\
\text { workers reporting } \\
\text { inadequate PPE }\end{array}$ \\
\hline General community & $3623 / 32980571$ & $0.33 \%$ & 1 (ref) & 1 (ref) & .. & .. \\
\hline \multicolumn{7}{|l|}{ Front-line health-care worker } \\
\hline Inpatient & $564 / 184293$ & $9 \cdot 18 \%$ & $23 \cdot 58(21 \cdot 20-26 \cdot 25)$ & $24.30(21.83-27.06)$ & $23 \cdot 7 \%$ & $11 \cdot 9 \%$ \\
\hline Nursing homes & $118 / 52901$ & $6 \cdot 69 \%$ & $16 \cdot 48(13 \cdot 60-19 \cdot 97)$ & $16 \cdot 24(13 \cdot 39-19 \cdot 70)$ & $15 \cdot 4 \%$ & $16 \cdot 9 \%$ \\
\hline Outpatient hospital clinics & $51 / 45217$ & $3.38 \%$ & $10 \cdot 75(8 \cdot 10-14 \cdot 27)$ & $11.21(8.44-14.89)$ & $16 \cdot 3 \%$ & $12 \cdot 2 \%$ \\
\hline Home health sites & $36 / 38642$ & $2 \cdot 79 \%$ & $7 \cdot 79(5 \cdot 58-10 \cdot 87)$ & $7.86(5.63-10 \cdot 98)$ & $14 \cdot 7 \%$ & $15 \cdot 9 \%$ \\
\hline Ambulatory clinics & $44 / 66408$ & $1.99 \%$ & $6 \cdot 64(4 \cdot 90-9 \cdot 01)$ & $6 \cdot 94(5 \cdot 12-9 \cdot 41)$ & $19 \cdot 3 \%$ & $11 \cdot 8 \%$ \\
\hline Other & $73 / 64310$ & $3.41 \%$ & $9.42(7.42-11.96)$ & $9 \cdot 52(7 \cdot 49-12 \cdot 08)$ & $12 \cdot 0 \%$ & $13 \cdot 8 \%$ \\
\hline \multicolumn{7}{|c|}{$\begin{array}{l}\text { Model was stratified by } 5 \text {-year age group, calendar date at study entry, and country and adjusted for sex (male or female), history of diabetes (yes or no), heart disease } \\
\text { (yes or no), lung disease (yes or no), kidney disease (yes or no), current smoking (yes or no), and body-mass index }\left(17 \cdot 0-19 \cdot 9 \mathrm{~kg} / \mathrm{m}^{2}, 20 \cdot 0-24 \cdot 9 \mathrm{~kg} / \mathrm{m}^{2}, 25 \cdot 0-29 \cdot 9 \mathrm{~kg} / \mathrm{m}^{2} \text {, }\right. \\
\text { and } \geq 30 \cdot 0 \mathrm{~kg} / \mathrm{m}^{2} \text { ). Ambulatory clinics include free-standing (non-hospital) primary care or specialty clinics and school-based clinics. PPE=personal protective equipment. }\end{array}$} \\
\hline
\end{tabular}


accounting for other risk factors. Post-hoc analyses showed that Black, Asian, and minority ethnic health-care workers are at especially high risk of SARS-CoV-2 infection, with at least a fivefold increased risk of COVID-19 compared with the non-Hispanic white general community. Among front-line health-care workers, reuse of PPE or inadequate PPE were each associated with a subsequent increased risk of COVID-19. Although healthcare workers caring for patients with COVID-19 who reported inadequate PPE had the highest risk of SARSCoV-2 infection, increased susceptibility to infection was evident even among those reporting adequate PPE. Frontline health-care workers who worked in inpatient settings (where providers most frequently reported PPE reuse) and nursing homes (where providers most frequently reported inadequate PPE) had the greatest risk. Non-white healthcare workers were disproportionately affected by scant PPE adequacy and more likely to work in clinical settings with greater exposure to patients with COVID-19.

Our findings could help provide greater context for previous cross-sectional reports from public health authorities suggesting $10-20 \%$ of SARS-CoV-2 infections occur among health-care workers. ${ }^{6,7}$ Our results offer individual-level data additionally accounting for workplace risk factors that complement these limited reports by providing a more precise assessment of the magnitude of increased risk among health-care workers during the initial phases of the COVID-19 pandemic. Taken in the context of the requirement for testing to establish a COVID-19 diagnosis, our range of results based on either reporting a positive test for COVID-19 or symptoms predictive of COVID-19 offer several complementary estimates for risk among front-line health-care workers.

We also provide evidence that sufficient availability of PPE, quality of PPE, or both reduce the risk of COVID-19, but reuse of PPE or inadequate PPE might confer comparably increased risk, which is compatible with findings from one of the first studies to specifically investigate PPE reuse. ${ }^{18}$ The greater risk associated with PPE reuse could be related to either self-contamination during repeated application and removal of PPE or breakdown of materials from extended wear. Of note, during the period of this study, disinfection protocols before PPE reuse were not widely available. ${ }^{11-13}$ Thus, results should be not extended to reflect risk of PPE reuse after such disinfection, which has now been implemented in various settings. An assessment of the PPE supply chain and equitable access to PPE should be a part of the deliberate and informed decision making about resource allocation.

However, even with adequate PPE, health-care workers who cared for patients with COVID-19 remained at increased risk, highlighting the importance of not only ensuring PPE quality and availability but also other aspects of appropriate use, including correct application and removal of PPE and clinical situation (practice location). Moreover, these data underscore the possibility for health-care workers to perpetuate infections or contribute to community spread, particularly when asymptomatic or mildly symptomatic, and justify calls to increase testing to reduce hospital-based transmission. ${ }^{5}$

Notably, we recorded a significant difference in risk for health-care workers in the UK compared with the USA. This discrepancy could be attributable to country-specific or region-specific variation in population density, socioeconomic deprivation, overall availability or quality of PPE, and type of health-care settings, and these findings require further investigation. Our results might also reflect differences in access to testing among health-care workers compared with the general community in the UK compared with the USA. However, in secondary analyses using inverse probability-weighted Cox modelling adjusting for the probability of receiving a test, we also found that health-care workers in the UK were at higher risk of reporting a positive test. Furthermore, health-care workers were at greater risk of developing symptoms predictive of eventual COVID-19, which does not reflect access to testing. Thus, the higher risk noted in the UK could reflect a higher infection rate because of differences in the quality and appropriate use of PPE across practice settings ${ }^{19}$ or country-specific differences in PPE recommendations for health-care workers or the general public (eg, cloth face coverings). ${ }^{20,21}$ Ideally, we would assess risk within a population that has undergone uniform screening. However, the current shortage of PCR-based testing kits does not make such an approach feasible but could justify targeted screening of front-line health-care workers. ${ }^{5,22}$ Future studies using serological testing to ascertain SARS-CoV-2 infection will require assessments of test performance and the ability to distinguish recent or active infection from past exposure.

Our results are supported by historical data during similar infectious disease outbreaks. Ebola virus has a basic reproduction number (ie, the number of new cases generated from one individual) comparable with that for SARS-CoV-2. During the Ebola virus disease crisis, health-care workers comprised $3.9 \%$ of all cases, 21-32-times greater than the general public. ${ }^{23}$ During the severe acute respiratory syndrome epidemic, health-care workers comprised $20-40 \%$ of cases, ${ }^{24-26}$ and inadequate PPE was associated with increased risk. ${ }^{26}$ The experience with influenza A virus subtype H1N1 reaffirmed the importance of $\mathrm{PPE},{ }^{27}$ with much higher infection rates among health-care workers in dedicated containment units. ${ }^{28}$

Our study has several strengths. First, we used a smartphone app to rapidly obtain prospective data from a large multinational cohort in real time, offering actionable risk estimates to inform the public health response to an ongoing pandemic. ${ }^{29}$ By recruiting participants through existing cohorts, ${ }^{30}$ our results provide proof of concept of the feasibility of leveraging existing infrastructure and engaged participants to address a key knowledge gap. Second, we obtained information from participants who 
For the HDRUK Innovation Gateway see https:// healthdatagateway.org

For the Coronavirus Pandemic Epidemiology Consortium see https://www.monganinstitute. org/cope-consortium

For data updates see https:// covid.joinzoe.com did not have a positive COVID-19 test, which offered an opportunity to prospectively assess risk factors with minimal recall bias. Third, our study design recorded initial onset of symptoms, which minimises biases related to capturing only severe cases through hospitalisation records or death reports. Finally, we gathered information on a wide range of known or suspected risk factors for COVID-19 generally not available in existing registries or population-scale surveillance efforts.

We acknowledge several limitations. First, details for some exposures were shortened to ensure our survey was brief. For example, we did not ask about specific occupations, experience level, type of PPE used (eg, masks, respirators, or powered air-purifying respirators), receipt of PPE training (eg, mask fit-testing or application and removal of PPE), and frequency of exposure to patients with SARS-CoV-2 infection or aerosolising procedures. We are pursuing additional questionnaires to more deeply investigate these topics in a subset of participants. Second, our findings are based on self-report. However, alternative exposure measures, such as PPE supply, or assessment of additional outcomes would have been difficult to obtain within the context of a fast-moving pandemic. In future studies, linkage to other sources (eg, electronic health records) might be possible. Third, our cohort is not a random sampling of the population. Although this limitation is inherent to any study requiring voluntary provision of information, we acknowledge that data collection through smartphone adoption has comparatively lower penetrance among some socioeconomic groups and adults older than age 65 years, despite smartphone use by $81 \%$ of US adults. ${ }^{31}$ This limitation could have resulted in selection bias, although our primary conclusions were robust to several sensitivity and secondary analyses. In future studies, we plan greater targeted outreach of underrepresented populations. Our primary outcome was based on the report of a positive COVID-19 test. During the study period, this outcome would generally reflect a positive PCR-based swab, which should be moderately specific, compared with antibody testing, which was not widely available. However, any misclassification of positive testing should be non-differential according to occupation.

In conclusion, we reported increased risk for SARS-CoV-2 infection among front-line health-care workers compared with the general community, using either self-reported data on COVID-19 testing positivity or a symptom-based predictor of positive infection status. This risk was especially high among Black, Asian, and minority ethnic health-care workers and individuals in direct contact with patients with COVID-19 who reported inadequate PPE availability or were required to reuse PPE. Ensuring the adequate allocation of PPE is important to alleviate structural inequities in COVID-19 risk. However, because infection risk was increased even with adequate PPE, our results suggest the need to ensure proper use of PPE and adherence to other infection control measures. Further intervention studies investigating modifiable risk factors for health-care worker-related SARS-CoV-2 infection, ideally accounting for differential exposure according to race and ethnic background and care location, are urgently needed to support our observational findings.

\section{Contributors}

LHN, DAD, MSG, JW, SO, CJS, TDS, and ATC contributed to study concept and design. LHN, DAD, MSG, JW, SO, RD, JC, CJS, TDS, and ATC contributed to acquisition of data. LHN, DAD, MSG, ADJ, C-GG, WM, RSM, DRS, C-HL, SK, and MS contributed to data analysis. LHN, DAD, and ATC contributed to initial drafting of the manuscript. All authors contributed to interpretation of data and critical revision of the report. SO, CJS, TDS, and ATC contributed to study supervision.

\section{Declaration of interests}

JW, RD, and JC are employees of Zoe Global. TDS is a consultant to Zoe Global. DAD and ATC previously served as investigators on a clinical trial of diet and lifestyle using a separate smartphone application that was supported by Zoe Global. All other authors declare no competing interests.

\section{Data sharing}

Data collected in the COVID-19 Symptom Study smartphone application are being shared with other health researchers through the UK National Health Service-funded Health Data Research UK (HDRUK) and Secure Anonymised Information Linkage consortium, housed in the UK Secure Research Platform (Swansea, UK). Anonymised data are available to be shared with HDRUK researchers according to their protocols in the public interest. US investigators are encouraged to coordinate data requests through the Coronavirus Pandemic Epidemiology Consortium. Data updates can be found online.

\section{Acknowledgments}

LHN is supported by the American Gastroenterological Association Research Scholars Award. DAD is supported by the National Institute of Diabetes and Digestive and Kidney Diseases (K01DK120742). ATC is the Stuart and Suzanne Steele Massachusetts General Hospital (MGH) Research Scholar and a Stand Up to Cancer scientist. National Institutes of Health grants related to this project include UM1 CA186107 (to AHE and MJS), U01 CA176726 (to AHE and WCW), U01 CA167552 (to WCW and LAM), U01 HL145386 (to JEC), R24 ES028521 (to JEC), P30ES000002 (to JEH), and grant contract 200-2017-M-94186 (to JEC). The

Massachusetts Consortium on Pathogen Readiness and Mark and Lisa Schwartz supported investigators from MGH. Investigators from King's College London were supported by the Wellcome Trust and Engineering and Physical Sciences Research Council (WT212904/Z/18/Z,

WT203148/Z/16/Z, T213038/Z/18/Z), the National Institute for Health Research Guy's and St Thomas' NHS Foundation Trust and King's College London Biomedical Research Centre, the UK Medical Research Council and British Heart Foundation (MR/M016560/1), UK Research and Innovation London Medical Imaging and Artificial Intelligence Centre for Value Based Healthcare, and the Alzheimer's Society (AS-JF-17-011). We thank all individuals who downloaded the COVID Symptom Study smartphone application (app), including participants of cohort studies within the COronavirus Pandemic Epidemiology (COPE) Consortium (appendix p 3). We thank investigators of cohort studies enrolled in the COPE Consortium for their assistance in disseminating the COVID Symptom Study smartphone app to their study participants, and the MGH Clinical and Translational Epidemiology Unit Clinical Research Coordination team. We thank Zoe Global for providing in-kind support for all aspects of building, running, and supporting the COVID-19 Symptom Study smartphone app and service to users worldwide. Finally, we thank Stand Up to Cancer for their assistance in media and social media outreach.

Editorial note: the Lancet Group takes a neutral position with respect to territorial claims in published maps and institutional affiliations.

References

1 Anderson RM, Heesterbeek H, Klinkenberg D, Hollingsworth TD. How will country-based mitigation measures influence the course of the COVID-19 epidemic? Lancet 2020; 395: 931-34. 
2 Johns Hopkins University of Medicine. Coronavirus resource center. July 22, 2020. https://coronavirus.jhu.edu/map.html (accessed July 22, 2020).

3 Kirby T. Evidence mounts on the disproportionate effect of COVID-19 on ethnic minorities. Lancet Respir Med 2020; 8: 547-48.

4 Garg S, Kim L, Whitaker M, et al. Hospitalization rates and characteristics of patients hospitalized with laboratory-confirmed coronavirus disease 2019: COVID-NET, 14 States, March 1-30, 2020. MMWR Morb Mortal Wkly Rep 2020; 69: 458-64.

5 Black JRM, Bailey C, Przewrocka J, Dijkstra KK, Swanton C. COVID-19: the case for health-care worker screening to prevent hospital transmission. Lancet 2020; 395: 1418-20.

6 CDC COVID-19 Response Team. Characteristics of health care personnel with COVID-19: United States, February 12-April 9, 2020. MMWR Morb Mortal Wkly Rep 2020; 69: 477-81.

7 Lazzerini M, Putoto G. COVID-19 in Italy: momentous decisions and many uncertainties. Lancet Glob Health 2020; 8: e641-42.

8 Verbeek JH, Rajamaki B, Ijaz S, et al. Personal protective equipment for preventing highly infectious diseases due to exposure to contaminated body fluids in healthcare staff. Cochrane Database Syst Rev 2020; 4: CD011621.

9 US Centers for Disease Control and Prevention. Interim infection prevention and control recommendations for healthcare personnel during the coronavirus disease 2019 (COVID-19) pandemic. July 9, 2020. https://www.cdc.gov/coronavirus/2019-ncov/hcp/ infection-control-recommendations.html (accessed July 15, 2020).

10 Public Health England. COVID-19 personal protective equipment (PPE): 5-summary of PPE recommendations for health and social care workers. June 18, 2020. https://www.gov.uk/government/ publications/wuhan-novel-coronavirus-infection-prevention-andcontrol/covid-19-personal-protective-equipment-ppe\#summary-ofppe-recommendations-for-health-and-social-care-workers (accessed July 15, 2020).

11 Fischer R, Morris DH, van Doremalen N, et al. Assessment of N95 respirator decontamination and re-use for SARS-CoV-2. medRxiv 2020; published online April 24. https://doi.org/10.1101/2020.04.11.20062018 (preprint).

12 Schwartz A, Stiegel M, Greeson N, et al. Decontamination and reuse of $\mathrm{N} 95$ respirators with hydrogen peroxide vapor to address worldwide personal protective equipment shortages during the SARS-CoV-2 (COVID-19) pandemic. Appl Biosaf 2020; 25: 67-70.

13 Livingston E, Desai A, Berkwits M. Sourcing personal protective equipment during the COVID-19 pandemic. JAMA 2020; 323: 1912-14.

14 The Lancet. COVID-19: protecting health-care workers. Lancet 2020; 395: 922.

15 Drew DA, Nguyen LH, Steves CJ, et al. Rapid implementation of mobile technology for real-time epidemiology of COVID-19. Science 2020; 368: 1362-67.
16 Rosner B. Fundamentals of biostatistics, 4th edn. Boston, MA, Brooks/Cole, 1995.

17 Menni C, Valdes AM, Freidin MB, et al. Real-time tracking of self-reported symptoms to predict potential COVID-19. Nat Med 2020; 26: 1037-40.

18 Chou R, Dana T, Buckley DI, Selph S, Fu R, Totten AM. Epidemiology of and risk factors for coronavirus infection in health care workers: a living rapid review. Ann Intern Med 2020; 173: $120-36$.

19 Kenber B, Lay K, Fisher L, Smith HL. Fifth of frontline doctors complain of unusable coronavirus PPE. The Times (London), May 8, 2020

20 Thomas JP, Srinivasan A, Wickramarachchi CS, Dhesi PK, Hung YM, Kamath AV. Evaluating the national PPE guidance for NHS healthcare workers during the COVID-19 pandemic. Clin Med 2020; 20: 242-47.

21 Cheng KK, Lam TH, Leung CC. Wearing face masks in the community during the COVID-19 pandemic: altruism and solidarity. Lancet 2020; published online April 16. https://doi.org/10.1016/S0140-6736(20)30918-1.

22 Treibel TA, Manisty C, Burton M, et al. COVID-19: PCR screening of asymptomatic health-care workers at London hospital. Lancet 2020; 395: 1608-10.

23 Houlihan CF, McGowan CR, Dicks S, et al. Ebola exposure, illness experience, and Ebola antibody prevalence in international responders to the West African Ebola epidemic 2014-2016: a cross-sectional study. PLoS Med 2017; 14: e1002300.

24 Koh D, Lim MK, Chia SE. SARS: health care work can be hazardous to health. Occup Med 2003; 53: 241-43.

25 Seto WH, Tsang D, Yung RW, et al. Effectiveness of precautions against droplets and contact in prevention of nosocomial transmission of severe acute respiratory syndrome (SARS). Lancet 2003; 361: 1519-20.

26 Lau JT, Fung KS, Wong TW, et al. SARS transmission among hospital workers in Hong Kong. Emerg Infect Dis 2004; 10: 280-86.

27 Marshall C, Kelso A, McBryde E, et al. Pandemic (H1N1) 2009 risk for frontline health care workers. Emerg Infect Dis 2011; 17: 1000-06.

28 Chen MI, Lee VJ, Barr I, et al. Risk factors for pandemic (H1N1) 2009 virus seroconversion among hospital staff, Singapore. Emerg Infect Dis 2010; 16: 1554-61.

29 Chan AT, Brownstein JS. Putting the public back in public health: surveying symptoms of Covid-19. N Engl J Med 2020; published online June 5. https://doi.org/10.1056/NEJMp2016259.

30 Chan AT, Drew DA, Nguyen LH, et al. The COronavirus Pandemic Epidemiology (COPE) Consortium: a call to action. Cancer Epidemiol Biomarkers Prev 2020; 29: 1283-89.

31 Pew Research Center for Internet \& Technology. Mobile fact sheet. June 12, 2020. https://www.pewresearch.org/internet/fact-sheet/ mobile/ (accessed July 15, 2020). 